\title{
Understanding uncertainty in medicine: concepts and implications in medical education
}

\author{
Kangmoon Kim and Young-Mee Lee \\ Department of Medical Education, Korea University College of Medicine, Seoul, Korea
}

In an era of high technology and low trust, acknowledging and coping with uncertainty is more crucial than ever. Medical uncertainty has been considered an innate feature of medicine and medical practice. An intolerance to uncertainty increases physicians' stress and the effects of burnout and may be a potential threat to patient safety. Understanding medical uncertainty and acquiring proper coping strategies has been regarded to be a core clinical competency for medical graduates and trainees. Integrating intuition and logic and creating a culture that acknowledges medical uncertainty could be suggested ways to teach medical uncertainty. In this article, the authors describe the concepts of medical uncertainty, its influences on physicians and on medical students toward medical decision making, the role of tolerance/intolerance to uncertainty, and proposed strategies to improve coping with medical uncertainty.

Key Words: Uncertainty, Decision making, Medical education, Competency-based education, Heuristics

\section{Introduction}

Medical practice is well known to be full of uncertainty [1]. Even experienced doctors face uncertainty, especially regarding complex co-morbid medical conditions that may hinder applications of existing medical evidences. Facing medical uncertainty is a naturally occurring problem that trainees have to deal with during their development process. However, inadequate ability to cope with medical uncertainty may unnecessarily distress medical trainees, and this could harm patients [2]. Physicians who are intolerant to uncertainty have a tendency to prescribe excessive amounts of diagnostic tests [3] and are reluctant to disclose their uncertainties to their patients when making decisions [4]. Inadequate management of uncertainty may cause unnecessary concern and distress to patients [5]. Medical students usually face medical uncertainty during their clerkship and experience it as a cause of major stress [6]. In addition, medical students who cannot tolerate uncertainty are known to exhibit negative attitudes toward underserved [7]. When residents are confronted with complex contextual factors, they feel uncertain during clinical reasoning [8]. Understanding and acknowledging medical uncertainty and acquiring proper coping strategies is regarded as one of the core clinical competencies for medical graduates and trainees in Scotland, Australia, European Union, United Kingdom, and United States [9-14]. In future
Received: May 22, 2018 • Revised: June 27, 2018 • Accepted: July 5, 2018 Corresponding Author: Young-Mee Lee (https://orcid.org/0000-0002-4685-9465) Department of Medical Education, Korea University College of Medicine, 73 Inchon-ro, Seongbuk-gu, Seoul 02841, Korea

Tel: +82.2.2286.1098 Fax: +82.2.928.1647 email: ymleehj@korea.ac.kr
Korean J Med Educ 2018 Sep; 30(3): 181-188.

https://doi.org/10.3946/kjme.2018.92

eISSN: 2005-7288

(C) The Korean Society of Medical Education. All rights reserved. This is an open-access article distributed under the terms of the Creative Commons Attribution Non-Commercial License (http:// creativecommons.org/licenses/by-nc/3.0/), which permits unrestricted non-commercial use, distribution, and reproduction in any medium, provided the original work is properly cited. 
medical practices, the application of high technology and precision medicine, including high-end information technology and artificial intelligence, may reduce uncertainty in medicine. However, novel knowledge and technology of the future, paradoxically, may bring up newer and unpredicted uncertainty in medical practice. Even in the era of the fourth industrial revolution, uncertainty in medicine may persist or even increase. Therefore, medical academics and training institutions should be able to help medical students and trainees better prepared to cope with uncertainty.

In this paper, the authors introduce the concept of medical uncertainty, its influence on physicians and medical students with regards to medical decision making, the role of tolerance/intolerance in uncertainty, and proposed strategies for managing medical uncertainty.

\section{Concepts and natures of medical un- certainty}

Osler [15] once mentioned, "medicine is science of uncertainty and art of probability.' Medical sociologist Fox [16] discerned that medical uncertainty in medicine comes from the limitations of medical knowledge, limitations of personal knowledge, and a difficulty distinguishing between the two. Since then, defining medical certainty has been attempted at various times [17-22].

Beresford described three sources of medical uncertainty: technical, personal, and conceptual [22]. Technical uncertainty arises from inadequate or incomplete scientific data and could be understood as data-related uncertainty. Personal uncertainty arises from an uncertain doctor-patient relationship. Conceptual uncertainty arises from a difficulty in applying data to real situations. Technical uncertainty could be considered first-order uncertainty and personal and conceptual uncertainty as second-order, or metauncertainty, as described by Savulescu [23]. First-order uncertainty is derived from uncertain probability of future outcomes and relates to scientific nature itself, whereas second-order uncertainty occurs when applying data to patient care, and it includes not only uncertainty that arises from real outcomes of given probabilities but also patients' desire or degree of interest regarding the outcome [23].

The terms 'uncertainty' and 'ambiguity' are sometimes used interchangeably [20]. Some researchers, such as Han et al. [19], believe ambiguity to be one of the sources of uncertainty. Han et al. [19] addressed various studies in literature from diverse disciplines, including psychology, communication, and health services, and suggested a new taxonomy of medical uncertainty consisting of three dimensions: sources, issues, and locus. The sources of uncertainty emerge from probability, ambiguity, and complexity. Probability refers to risk, and it comes from the indeterminacy of future outcomes. Ambiguity refers to indecisiveness, and it arises from imprecision, conflicting information, and a lack of evidence. Complexity refers to incomprehensibility, and it arises from a multiplicity of causal factors or difficulty of interpretation.

\section{Medical uncertainty and clinical rea- soning}

Clinical reasoning is a thinking process performed in medical practice by healthcare professionals. It forms the core of professional autonomy [24]. One of the critical components involving clinical decision making is diagnostic reasoning. However, it has been reported that 
the accuracy of physicians' diagnosis, as assessed by autopsies, has not improved since the early 20th century $[25,26]$. As uncertainty lies in diagnosis and treatment, which are the major components of clinical decision making [27], the perception of uncertainty by physicians during clinical reasoning would impose a great deal of significance on patient care.

When confronted with uncertainties in decision making, physicians often deny uncertainty and uphold medical orthodoxy, merely adopt the practice of other physicians, or use heuristics [28,29]. Heuristics are a method that could easily be applied when systemic or rational judgement is not required or cannot be attained [28]. Hall [28] described common heuristics and biases that physicians face during the process of clinical reasoning: representative heuristic, availability heuristic, anchoring and adjustment heuristic, hindsight bias, framing effect, optimistic bias, regret bias, socio-cultural bias, and others. These cognitive biases could be sources of errors in the process of clinical reasoning. Hall [28] warned of the potential harm of heuristic decision making without proper recognition of uncertainty. To help young doctors avoid errors derived from mere heuristic decision making, training recognition, admittance of unavoidable medical uncertainty, and coping strategies are needed.

The suggestion has been made that establishing and adapting to clinical guidelines are productive ways of responding to uncertainty in medicine [28]. Indeed, physicians tend to respond by adhering to guidelines when faced with uncertainty. However, despite the use of guidelines, uncertainties in medical practice are unlikely to be overcome [30]. Eddy [29] pointed out that some aspects of medical knowledge can never be verified by randomized controlled trial and uncertainty cannot be completely eliminated due to the variance of human nature. Besides, medical uncertainty, which can be resolved by medical knowledge and research evidence, is confined to first-order, or technical uncertainty [22,28]. Physicians still have to deal with second-order or meta-uncertainty in clinical decision making, despite enormous clinical evidence and highly sophisticated technical data [28].

\section{Tolerance/intolerance of medical un- certainty}

\section{Demographic features related to tolerance of uncertainty}

Physicians' tolerance to uncertainty or ambiguity has been shown to differ by age, sex, and specialties. Geller et al. [17] reported that psychiatrists, and physicians who graduated medical school when they were older, who were open to prescribing novel predictive tests, had higher tolerance to ambiguity. Gerrity et al. [18] showed that women, surgeons, general practitioners, or physicians who were practicing for shorter periods tended to experience more stress from uncertainty. Similarly, in a study using Budner's Intolerance of Ambiguity Scale, surgeons were significantly less tolerant to uncertainty than physicians [31].

\section{Negative influences of intolerance to me- dical uncertainty}

Previous studies have shown that intolerance to medical uncertainty can negatively affect doctors' emotions, mental health, and job satisfaction. Physicians who experienced more stress from uncertainty had higher levels of burnout [32] and experienced increased work-related stress [33] and decreased job satisfaction [34]. Cooke et al. [35] showed that a general intolerance to uncertainty, anxiety due to clinical uncertainty, and 
the reluctance to disclose uncertainty to patients have been related to a higher risk of burnout. In contrast, the general practice registrars who had higher scores on the resilience scale had less burnout, less anxiety, and tolerance to uncertainty. Additionally, physician's anxiety provoked by uncertainty seemed to be associated with increased patient hospital charges [36] and more referrals [32]. Intolerance to uncertainty could be related to discomfort with elderly patients and patients who have psychological problems [18]. The impact of uncertainty-related anxiety on work-related satisfaction was more prominent for training physicians [34]. Residents who had less tolerance to uncertainty experienced more anxiety when caring for dying patients [37]. Residents who had higher levels of stress from uncertainty had lower levels of resilience scores and higher levels of burnout and depression [38].

Why is uncertainty stressing out doctors? Ghosh [1] proposed that a physician who is intolerant to uncertainty seems to perceive uncertainty as a threat. To reduce burnout or distress from medical uncertainty and to transform the cognitive dissonance provoked by clinical uncertainty to a self-directed learning moment, medical students and trainees should be aware of and taught how to lower perceived threats from uncertainty while improving resilience.

\section{Coping with medical uncertainty}

Studies regarding coping mechanisms and strategies to improve dealing with medical uncertainty have been conducted. The previous studies mostly focused on behavioural responses to uncertainty: (1) positive behavioural responses, such as approach, action, decision making, and information seeking; and (2) negative behavioural responses, such as avoidance, inaction, decision deferral, or inattention [21]. Hillen et al. [21] classified uncertainty responses as positive or negative and proposed an integrative model that includes responses to uncertainty. Han et al. [19] claimed that uncertainty could be considered a perception or metacognition, namely 'knowing of unknowing.'

Generally, medical trainees resolve medical uncertainty by consulting seniors through a predetermined hierarchy [39]. Farnan et al. [2] reported that residents had managed uncertainty in critical incidents through a firm hierarchy, seeking help from colleagues and literature first, and then senior residents, specialty fellows, and the attending physician. In this study, the reported barriers to contact seniors included (1) fear of losing autonomy in decision making; (2) fear of revealing knowledge gaps; (3) a tendency to adhere to the defined hierarchy; and (4) concern about repercussions. Friedman et al. [40] studied residents' behaviours to resolve uncertainty and suggested that mere perception of firm hierarchy could be a barrier to early action to resolve uncertainty.

Cristancho et al. [41] have categorized surgeons' responses to uncertainty as prioritizing alternatives, re-evaluating the plan, creating novel solutions, and seeking advice. The authors reported that the level of tolerance to uncertainty improved over time and experience via training. Similar results have been reported in studies involving family medicine residents, students, and attending physicians $[42,43]$.

Ghosh [44] recommends some behavioural strategies to decrease clinical uncertainty: trying to apply the best available evidence-based information, taking careful note of the patients' history, excluding fatal diagnoses, and encouraging patients to take part in decisionmaking. These actions are the principal components that should be taught and learned during medical training. However, as discussed earlier, medical uncertainty cannot be eliminated completely in real world situations. 


\section{Teaching strategies to manage medical uncertainty}

Geller et al. [17] have reported that tolerance to ambiguity does not change throughout medical school and argue that it should be included as one of the qualifications required for admittance to medical school [45]. Meanwhile, some researchers claim that tolerance to uncertainty in medical students may change or improve during medical school, at least in part $[6,46]$.

Despite the nature of uncertainty of medicine and medical practice, the culture of medicine does not seem to admit it [47]. Domen [48] argues that embracing uncertainty could improve personal well-being, construct effective mentorship, establish a culture of respect throughout the institution, bring out meaningful learning, and enhance sound doctor-patient relationships and patient-centred care.

Ghosh [1] proposes some techniques to decrease uncertainty in clinical practice [1]. Physicians must be clear when addressing patients that they are ready to answer any questions and ought to introduce variable sources of information about health. When asked about things that are uncertain, physicians should acknowledge their ignorance and try to seek an answer. Physicians should listen to patients with sympathy and have patients become involved in the decision-making process, with explanations of test results, known risks, and options for treatment using easy language, while admitting the possibility of biases and errors in the process of clinical reasoning.

Teaching dual process theory has been reported to somewhat improve accuracy in clinical reasoning rather than merely teaching about errors and biases using heuristics in uncertain situations [49]. Adopted from cognitive psychological theories, Croskerry [50] sug- gested an integration of the two systems of decision making: system 1 (heuristic, intuitive) and system 2 (systematic, analytical). System 1 is a hardwiring system that is passive and automatic. It is based on intuitive thinking or heuristics, which are provoked by pattern recognition. This can be influenced by contexts such as age, gender, emotion, and experience. On the other hand, system 2 is basically logical and reasonable. This can be learned from education and training. System 1 is used widely throughout everyday life, and it could be an effective and powerful tool in simple, routine clinical situations. For example, a physician will immediately diagnose acute pharyngo-tonsillitis (APT) by observing a throat that has swollen, reddish, ulcerative tonsils. Furthermore, the physician could consider the patient's sex, age, and the presence of signs such as fever and lethargy or other symptoms such as cough and rhinorrhoea. But generally, he or she would not meticulously consider the prevalence of APT in the district or the sensitivity and specificity of the visual examination of the throat deliberately. Taking the prevalence of a disease and sensitivity or specificity of an examination or test into account could be considered a system 2 process of decision making [50]. This could be adopted in rather complex situations [51]. Teaching to be mindful of integrated processes of reasoning in daily clinical practice could be a useful way of teaching strategies to cope with uncertainty in medicine [27]. Further studies are needed to examine the effect of cognitive intervention on teaching dual process theory in large populations and its effect on uncertainty tolerance.

\section{Conclusion}

Despite dealing with medical uncertainty, which is recognized as one of the core competencies in medical 
education, this area still remains unknown and unexplored in medical education. There is not much evidence on the ways of effective teaching and learning with regards to medical uncertainty, and improving tolerance towards it. Considering it does not have a negligible effect on medical practice, more attention and research on this issue is needed.

Creating a culture that acknowledges medical uncertainty would be the first step to teach medical uncertainty and tolerance towards it. Dual process theory adopted from cognitive decisional science sheds some light on teaching strategies to cope with medical uncertainty. Further studies are needed to examine the effect of cognitive intervention regarding dual process theory in large populations and its effect on uncertainty tolerance.

\section{ORCID:}

Kangmoon Kim: https://orcid.org/0000-0002-3518-575X; Young-Mee Lee: https://orcid.org/0000-0002-4685-9465

Acknowledgements: None.

Funding: This work was supported by the National Research Foundation of Korea grant funded by the Korea government (Ministry of Science, ICT and Future Planning) (no., 2016R1A2B4013614).

Conflicts of interest: No potential conflict of interest relevant to this article was reported.

Author contributions: Drafting the article: KMK; critical revision of the article: YML; and final approval of the version to be published: YML.

\section{References}

1. Ghosh AK. Understanding medical uncertainty: a primer for physicians. J Assoc Physicians India. 2004;52:739-
742.

2. Farnan JM, Johnson JK, Meltzer DO, Humphrey HJ, Arora VM. Resident uncertainty in clinical decision making and impact on patient care: a qualitative study. Qual Saf Health Care. 2008;17(2):122-126.

3. Kassirer JP, Kopelman RI. Tolerating uncertainty. Hosp Pract (Off Ed). 1987;22(4):21-22,27-28.

4. Katz J. Why doctors don't disclose uncertainty. Hastings Cent Rep. 1984;14(1):35-44.

5. Smith AK, White DB, Arnold RM. Uncertainty: the other side of prognosis. N Engl J Med. 2013;368(26): 2448-2450.

6. Nevalainen MK, Mantyranta T, Pitkala KH. Facing uncertainty as a medical student: a qualitative study of their reflective learning diaries and writings on specific themes during the first clinical year. Patient Educ Couns. 2010;78(2):218-223.

7. Wayne S, Dellmore D, Serna L, Jerabek R, Timm C, Kalishman S. The association between intolerance of ambiguity and decline in medical students' attitudes toward the underserved. Acad Med. 2011;86(7):877882.

8. McBee E, Ratcliffe T, Picho K, et al. Consequences of contextual factors on clinical reasoning in resident physicians. Adv Health Sci Educ Theory Pract. 2015; 20(5):1225-1236.

9. Harden RM, Crosby JR, Davis MH, Friedman M. AMEE guide no. 14: outcome-based education: part 5-from competency to meta-competency: a model for the specification of learning outcomes. Med Teach. 1999; $21(6): 546-552$.

10. Englander R, Cameron T, Ballard AJ, Dodge J, Bull J, Aschenbrener CA. Toward a common taxonomy of competency domains for the health professions and competencies for physicians. Acad Med. 2013;88(8): 1088-1094.

11. Simpson JG, Furnace J, Crosby J, et al. The Scottish 
doctor: learning outcomes for the medical undergraduate in Scotland: a foundation for competent and reflective practitioners. Med Teach. 2002;24(2):136-143.

12. General Medical Council. Outcomes for graduates (Tomorrow's Doctors). Manchester, UK: General Medical Council; 2015.

13. Cumming A, Ross M. Learning outcomes/competencies for undergraduate medical education in Europe: Tuning Project (Medicine). http://www.unideusto.org/tuningeu/ images/stories/Summary_of_outcomes_TN/Learning_Out comes_Competences_for_Undergraduate_Medical_Educa tion_in_Europe.pdf. Published 2013. Accessed April 22, 2018.

14. Expectations for level of achievement of the graduate capabilities in each phase of the curriculum. Sydney, Australia: The University of New South Wales; 2011.

15. Osler W, Bean RB, Bean WB. Aphorisms from his bedside teachings and writings. Springfield, USA: Charles C. Thomas; 1961.

16. Fox RC. The evolution of medical uncertainty. Milbank Mem Fund Q Health Soc. 1980;58(1):1-49.

17. Geller G, Tambor ES, Chase GA, Holtzman NA. Measuring physicians' tolerance for ambiguity and its relationship to their reported practices regarding genetic testing. Med Care. 1993;31(11):989-1001.

18. Gerrity MS, DeVellis RF, Earp JA. Physicians' reactions to uncertainty in patient care: a new measure and new insights. Med Care. 1990;28(8):724-736.

19. Han PK, Klein WM, Arora NK. Varieties of uncertainty in health care: a conceptual taxonomy. Med Decis Making. 201 1;31(6):828-838.

20. Hancock J, Roberts M, Monrouxe L, Mattick K. Medical student and junior doctors' tolerance of ambiguity: development of a new scale. Adv Health Sci Educ Theory Pract. 2015;20(1):113-130.

21. Hillen MA, Gutheil CM, Strout TD, Smets EM, Han PK. Tolerance of uncertainty: conceptual analysis, integrative model, and implications for healthcare. Soc Sci Med. 2017;180:62-75.

22. Beresford EB. Uncertainty and the shaping of medical decisions. Hastings Cent Rep. 1991;21(4):6-11.

23. Savulescu J. Treatment limitation decisions under uncertainty: the value of subsequent euthanasia. Bioethics. 1994;8(1):49-73.

24. Higgs J, Jones M. Clinical reasoning in the health professionals. 2nd ed. Oxford, UK: Butterworth Heinemann; 2002.

25. Lundberg GD. Low-tech autopsies in the era of high-tech medicine: continued value for quality assurance and patient safety. JAMA. 1998;280(14):1273-1274.

26. Kuijpers CC, Fronczek J, van de Goot FR, Niessen HW, van Diest PJ, Jiwa M. The value of autopsies in the era of high-tech medicine: discrepant findings persist. J Clin Pathol. 2014;67(6):512-519.

27. Croskerry P. From mindless to mindful practice: cognitive bias and clinical decision making. N Engl J Med. 2013;368(26):2445-2448.

28. Hall KH. Reviewing intuitive decision-making and uncertainty: the implications for medical education. Med Educ. 2002;36(3):216-224.

29. Eddy DM. Variations in physician practice: the role of uncertainty. Health Aff (Millwood). 1984;3(2):74-89.

30. Mehlman MJ. Medical practice guidelines as malpractice safe harbors: illusion or deceit? J Law Med Ethics. 2012;40(2):286-300.

31. McCulloch P, Kaul A, Wagstaff GF, Wheatcroft J. Tolerance of uncertainty, extroversion, neuroticism and attitudes to randomized controlled trials among surgeons and physicians. Br J Surg. 2005;92(10):1293-1297.

32. Bachman KH, Freeborn DK. HMO physicians' use of referrals. Soc Sci Med. 1999;48(4):547-557.

33. Iannello P, Mottini A, Tirelli S, Riva S, Antonietti A. Ambiguity and uncertainty tolerance, need for cognition, and their association with stress: a study among Italian 
practicing physicians. Med Educ Online. 2017;22(1): 1270009 .

34. Bovier PA, Perneger TV. Stress from uncertainty from graduation to retirement: a population-based study of Swiss physicians. J Gen Intern Med. 2007;22(5):632638.

35. Cooke GP, Doust JA, Steele MC. A survey of resilience, burnout, and tolerance of uncertainty in Australian general practice registrars. BMC Med Educ. 2013;13:2.

36. Allison JJ, Kiefe CI, Cook EF, Gerrity MS, Orav EJ, Centor R. The association of physician attitudes about uncertainty and risk taking with resource use in a Medicare HMO. Med Decis Making. 1998;18(3):320329.

37. Kvale J, Berg L, Groff JY, Lange G. Factors associated with residents' attitudes toward dying patients. Fam Med. 1999;31(10):691-696.

38. Simpkin AL, Khan A, West DC, et al. Stress from uncertainty and resilience among depressed and burned out residents: a cross-sectional study. Acad Pediatr. 2018 Mar 7 [Epub]. https://doi.org/10.1016/j.acap.2018.03.002.

39. Hamui-Sutton A, Vives-Varela T, Gutiérrez-Barreto S, Leenen I, Sánchez-Mendiola M. A typology of uncertainty derived from an analysis of critical incidents in medical residents: a mixed methods study. BMC Med Educ. 2015;15:198.

40. Friedman Z, Hayter MA, Everett TC, Matava CT, Noble LM, Bould MD. Power and conflict: the effect of a superior's interpersonal behaviour on trainees' ability to challenge authority during a simulated airway emergency. Anaesthesia. 2015;70(10):1119-1129.

41. Cristancho SM, Apramian T, Vanstone M, Lingard L, Ott M, Novick RJ. Understanding clinical uncertainty: what is going on when experienced surgeons are not sure what to do? Acad Med. 2013;88(10):1516-1521.

42. Ledford CJ, Cafferty LA, Seehusen DA. Socializing identity through practice: a mixed methods approach to family medicine resident perspectives on uncertainty. Fam Med. 2015;47(7):549-553.

43. Simpson DE, Dalgaard KA, O'Brien DK. Student and faculty assumptions about the nature of uncertainty in medicine and medical education. J Fam Pract. 1986; 23(5):468-472.

44. Ghosh AK. Dealing with medical uncertainty: a physician's perspective. Minn Med. 2004;87(10):48-51.

45. Geller G. Tolerance for ambiguity: an ethics-based criterion for medical student selection. Acad Med. 2013; 88(5):581-584.

46. Han PK, Schupack D, Daggett S, Holt CT, Strout TD. Temporal changes in tolerance of uncertainty among medical students: insights from an exploratory study. Med Educ Online. 2015;20:28285.

47. Luther VP, Crandall SJ. Commentary: ambiguity and uncertainty: neglected elements of medical education curricula? Acad Med. 2011;86(7):799-800.

48. Domen RE. The ethics of ambiguity: rethinking the role and importance of uncertainty in medical education and practice. Acad Pathol. 2016;3:2374289516654712.

49. Eva KW, Hatala RM, Leblanc VR, Brooks LR. Teaching from the clinical reasoning literature: combined reasoning strategies help novice diagnosticians overcome misleading information. Med Educ. 2007;41(12):1152-1158.

50. Croskerry P. A universal model of diagnostic reasoning. Acad Med. 2009;84(8):1022-1028.

51. Mamede S, Schmidt HG, Rikers R. Diagnostic errors and reflective practice in medicine. J Eval Clin Pract. 2007;13(1):138-145. 